'A Lucht, ${ }^{2} \mathrm{M}$ Shah, ${ }^{3,4} \mathrm{M}$ Kretzschmar. 'Labor Krone, Bad Salzuflen, Germany; 2Johns Hopkins University, Division of Infectious Diseases, Baltimore, MD, United States; ${ }^{3}$ University Medical Centre Utrecht, Julius Centre for Health Sciences \& Primary Care, Utrecht, The Netherlands; ${ }^{4}$ Centre for Infectious Diseases Control, RIVM, Bilthoven, The Netherlands

Background A frequently used HIV risk reduction method among men who have sex with men (MSM) is serosorting, which can be defined as a restriction of sexual partnerships to those who are of the same HIV serostatus. This partner selection strategy has been shown to reduce HIV transmission in epidemiological studies and mathematical models, but may lead to acquisition of other sexually transmitted infections (STI) including syphilis. We sought to evaluate the impact of HIV serosorting on syphilis prevalence, and to assess whether serosorting could account for an observed rise in syphilis incidence among MSM in San Francisco.

Methods A deterministic SIRS (susceptible-infectious-resistantsusceptible) model of syphilis transmission among HIV-negative and HIV-positive MSM was developed; model input parameters were based on epidemiological data from San Francisco between 1998 and 2004. The primary outcome was the impact of HIV serosorting on syphilis prevalence; we further evaluated the influence of HIV prevalence and average number of sexual partnerships on this serosorting effect.

Results Simulations showed that for base-case conditions, HIV serosorting increases syphilis transmission among HIV-positive and also among HIV-negative MSM so that syphilis could become endemic like in San Francisco. Only under very specific circumstances with high levels of serosorting among HIV-negative men can serosorting decrease syphilis prevalence. The size of the impact of serosorting on syphilis prevalence depends on HIV prevalence and partnership number

Conclusions Our mathematical model adds evidence to the conclusion from an earlier ecological study suggesting that serosorting of HIV-negative and HIV-positive MSM may explain the increased syphilis incidence observed in San Francisco between 1998 and 2004. Our model results may have important implications for MSM not only in the US. Public health recommendations on HIV serosorting as an HIV harm reduction strategy should take into account the potential unintended consequence of increasing the prevalence of other STIs.

\section{P3.133 POTENTIAL HIV TRANSMISSIONS FROM INFECTED DRUG INJECTORS AND MEN WHO HAVE SEX WITH MEN IN SAINT PETERSBURG, RUSSIA}

doi:10.1136/sextrans-2013-051184.0592

'E W White, ${ }^{1} \mathrm{~L}$ Niccolai, ${ }^{2} \mathrm{O}$ V Toussova, ${ }^{2} \mathrm{~S}$ V Verevochkin, ${ }^{2} \mathrm{~A}$ P Kozlov, ${ }^{1} \mathrm{R}$ Heimer. ${ }^{1}$ Yale School of Public Health, New Haven, CT, United States; ${ }^{2}$ The Biomedical Center, Saint Petersburg, Russian Federation

Background Less is known about HIV prevalence and incidence among men who have sex with men (MSM) in Saint Petersburg, Russia than in other large European cities, but it is clear that in that city the public health impact of HIV on drug injectors and their sex partners is remarkably high. We estimated the public health impact Methods Respondent-driven sampling (RDS) yielded behavioural and HIV testing data and specimens for HIV antibody testing from 201 MSM who did not report injecting drugs and 691 drug injectors who did not report male-male sex. We estimated the potential for sexual HIV transmission from MSM and injectors unaware of their HIV infection, comparing unsafe encounters.

Results One quarter of people who inject drugs and 5\% of MSM were HIV infected and unaware of their infection. Based on our prior estimate that there were 83,100 drug injectors living in Saint Petersburg; about 20,000 injectors are unaware of their HIV infection. If $1 \%$ of the general population are MSM, we estimate that there are about 3,000 MSM with undiagnosed HIV infection in Saint Petersburg. The 169 injectors with undiagnosed infection reported unprotected sex with 37 partners who themselves injected drugs, and that the proportion of injectors who were uninfected was $56 \%$. Injectors with undiagnosed infection reported unprotected sex with 26 partners who did not inject drugs; the uninfected proportion of non-injecting partners of drug injectors was $83 \%$. Therefore, undiagnosed, HIV+ injectors exposed an estimated mean 0.25 uninfected partners in six months time. Those injectors expose 5,821 (95\% CI 3,092-11,095) uninfected sex partners during a six-month period, whereas the 3,000 MSM with undiagnosed HIV infection in Saint Petersburg expose 4,800 (95\% CI 4,320-12,000).

Conclusion New sexual infections eminating from MSM may become comparable to those from drug injectors.

\section{P3.134 THE AGE-SPECIFIC DISTRIBUTION OF GENITAL HUMAN PAPILLOMAVIRUS (HPV) INFECTION AND HERPES SIMPLEX VIRUS-2 (HSV-2) ANTIBODIES AMONG MEN WITH GENDER- FIXED AND GENDER-FLUID SEXUAL BEHAVIOUR: THE HIM STUDY}

doi:10.1136/sextrans-2013-051184.0593

${ }^{1}$ A G Nyitray, 'M W Ross, ${ }^{1} \mathrm{M}$ Wilkerson, ${ }^{2} \mathrm{~L}$ L Villa, ${ }^{3} \mathrm{M}$ Abrahamsen, ${ }^{3} \mathrm{M}$ Papenfuss, ${ }^{4}$ E Lazcano-Ponce, ${ }^{3}$ A R Giuliano. 'University of Texas School of Public Health, Houston, TX, United States; ${ }^{2}$ School of Medicine, University of São Paulo, São Paulo, Brazil; ${ }^{3} \mathrm{H}$. Lee Moffitt Cancer Center and Research Institute, Tampa, FL, United States; ${ }^{4}$ Instituto Nacional de Salud Publica, Cuernavaca, Mexico

Background We investigated the age-specific distribution of genital HPV and HSV-2 antibodies among men whose sexual behaviour was confined either to men or to women (fixed) vs men whose sexual behaviour included both sexes (fluid). We hypothesised that fluid men may have more exposure to viral STIs.

Methods A total 4123 men from São Paulo, Brazil, Cuernavaca, Mexico, and Tampa, USA, aged 18-70 years, reported their sexual behaviour every six months for 2 years. Analysis was restricted to 1412 Brazilian men where fluidity had a much higher prevalence. Genital exfoliated cells for HPV PCR genotyping were collected every 6 months and blood for HSV-2 antibody testing was collected every 12 months. We used chi square and the Cochran-Armitage test to assess associations and age-specific linear trends.

Results Of 1292 men at enrollment who provided sexual behaviour information at $\geq 2$ time points, those aged 17-24 reported the most fluidity (24\%) and those aged 50-70 reported the least (16\%) although the linear trend was not significant $(p=0.12)$. After two years of follow up, HPV prevalence was comparable and stable by age among both fixed and fluid men for any HPV type, oncogenic types, and nononcogenic types. Prevalence of HSV-2 antibodies was higher among fluid than fixed men $(65 \%$ and $59 \%$, respectively) although not significantly different $(p=0.06)$. The prevalence of HSV-2 antibodies increased by age among both fixed and fluid men ( $p$ for trend $<0.0001$ and $=0.0006$, respectively).

Conclusions These data suggest that fluidity is not associated with increased prevalence of HPV and HSV-2. There was no trend for genital HPV by age which might be expected of a highly prevalent viral infection that often escapes immune surveillance; however, age was associated with HSV-2 antibodies as would be expected of a highly prevalent viral infection that less often escapes immune recognition.

\section{P3.135 HIGH PREVALENCE, INCIDENCE AND CLEARANCE OF ANAL HIGH-GRADE SQUAMOUS INTRAEPITHELIAL LESIONS (HSIL): EARLY EVIDENCE FROM A NATURAL HISTORY STUDY IN HOMOSEXUAL MEN}

doi:10.1136/sextrans-2013-051184.059 
'A E Grulich, II M Poynten, ${ }^{2} \mathrm{~J}$ Roberts, ${ }^{2} \mathrm{~A}$ Farnsworth, ${ }^{3,1} \mathrm{R} \mathrm{J}$ Hillman, ${ }^{1,4} \mathrm{D} \mathrm{J}$ Templeton, ${ }^{5} \mathrm{~S} N$ Tabrizi, ${ }^{5} \mathrm{~S} M \mathrm{M}$ Garland, ${ }^{6} \mathrm{C}$ K Fairley, ${ }^{1} \mathrm{~F}$ Jin. ${ }^{1}$ Kirby Institute, UNSW, Sydney, NSW, Australia; ${ }^{2}$ Douglass Hanly Moir Pathology, Sydney, NSW, Australia; ${ }^{3}$ Western Sydney Sexual Health Centre, University of Sydney, Sydney, NSW, Australia; "RPA Sexual Health, Royal Prince Alfred Hospital, Sydney, Australia; ${ }^{5}$ Royal Women's Hospital, University of Melbourne, Melbourne, Vic, Australia; ${ }^{\circ}$ Melbourne Sexual Health Centre, Melbourne, Vic, Australia

Background Homosexual men have high rates of anal cancer but an understanding of the epidemiology of HSIL, the presumed precursor, is lacking. We aimed to describe the epidemiology of anal HSIL, and association with human papillomavirus (HPV), in a community-recruited cohort of homosexual men.

Methods The Study of the Prevention of Anal Cancer is a threeyear prospective study of anal HPV infection and cancer precursors in homosexual men aged $\geq 35$. At each visit all men receive an anal swab for cytology and HPV genotyping (Roche Linear Array), and high resolution anoscopy with biopsy of suspected lesions. Anal HSIL was defined as having either intraepithelial neoplasia grade $2 / 3$ on histology and/or HSIL on cytology.

Results 293 men were recruited by December 2012. Median age was 49 and $28.3 \%$ were HIV-positive. The baseline prevalence of anal HSIL was $44.6 \%$ and $34.8 \%$ in the HIV-positive and -negative respectively $(p=0.119)$. Among those without HSIL at baseline, HSIL incidence was 30.0 and 20.0 per 100 person-years in the HIVpositive and -negative $(p=0.467)$. Among those with HSIL at baseline, the clearance rate was 37.0 and 44.1 per 100 person-years in the HIV-positive and -negative $(p=0.771)$. Men who tested HPV16 positive on their anal swab at baseline were more likely to develop incident HSIL (58.1 vs 16.1 per 100 person-years, $p=0.002$ ), and less likely to clear prevalent HSIL (15.9 vs. 65.9 per 100 person-years, $\mathrm{p}=0.006$ ).

Conclusion Anal HSIL were highly prevalent. Incidence and clearance were common and closely associated with HPV16 status. The high rate of clearance is consistent with the observation that anal HSIL progress to cancer less commonly than do cervical HSIL. The close association of persistence with HPV16 status suggests that HPV testing should be investigated as a means of identifying those with HSIL who are at highest risk of developing cancer.

\section{P3.136 EPIDEMIC OF LYMPHOGRANULOMA VENEREUM (LGV) IN EUROPE}

doi:10.1136/sextrans-2013-051184.0595

K Haar, G Spiteri, 0 Sfetcu, M van de Laar. European Centre for Disease Prevention and Control (ECDC), Stockholm, Sweden

Background Since 2003, outbreaks of lymphogranuloma venereum (LGV), caused by Chlamydia trachomatis genotypes L1-L3, have been described in men who have sex with men (MSM) in Europe. Awareness increased and enhanced surveillance systems were implemented and several countries in the European Union (EU/ EEA) reported high numbers of LGV cases.

Methods Surveillance for chlamydia, including LGV, in EU/EEA is coordinated by the European Centre for Disease Prevention and Control (ECDC). Since 2009, outbreak information across EU/ EEA is shared via the Epidemic Intelligence Information System for STI (EPIS STI) hosted by ECDC. Data reported by Member States covering the period 2009-2012 were analysed. Individual countries were invited to update detailed information on LGV cases.

Results In 2009-2011, 1642 cases of LGV were reported by 7 Member States: 1630 in men (61\% MSM). Median age was 39 years (range: 18 - 69). Overall, 909 LGV cases (55\%) were HIV-positive. Through EPIS STI, 279 male and 2 female cases from additional 6 countries were reported, mainly in capital areas. Symptoms were reported for 132 cases: 105 cases with and 27 without symptoms.
Proctitis was most commonly reported in men. Among those with known HIV status, $80 \%$ were HIV positive. $45 \%$ of cases in Spain were in migrants and $20 \%$ of the UK cases reported sexual contacts abroad.

Conclusions LGV remains to be primarily found in HIV positive MSM. Asymptomatic presentation hampers case detection and screening and routine genotyping among MSM were suggested to reduce further transmission of LGV. Screening practises differ across countries which results in diversity in number and nature of reported cases. Countries reported individual and clustered LGV cases in EPIS STI with more detailed information than in routine surveillance reports to ECDC. Efforts were made to increase European collaboration to get a better understanding of the evolving LGV epidemic in Europe.

\section{P3.137 RECTAL LUBRICANT USE \& INCIDENT STI INFECTIONS AT 9 US STD CLINICS}

doi:10.1136/sextrans-2013-051184.0596

1.2P M Gorbach, ${ }^{3} \mathrm{D}$ J Feaster, ${ }^{1} \mathrm{H}$ J Pines, ${ }^{3} \mathrm{Z}$ Gomez, ${ }^{4} \mathrm{~J}$ Castro, ${ }^{5} \mathrm{R}$ Bolan, ${ }^{6} \mathrm{~S}$ Henn, ${ }^{7}$ A Douaihy, ${ }^{8} \mathrm{M}$ Golden, ${ }^{3,9} \mathrm{~L}$ Metsch. ${ }^{1}$ University of California, Los Angeles, Los Angeles, CA, United States; 'David Geffen School of Medicine, Los Angeles, CA, United States; ${ }^{3}$ Miller School of Medicine, University of Miami, Miami, FL, United States; ${ }^{4}$ University of Miami, Miami, FL, United States; ${ }^{5}$ Los Angeles Gay and Lesbian Center, Los Angeles, CA, United States; ${ }^{6}$ Whitman-Walker Health, Washington, DC, DC, United States; 'University of Pittsburgh School of Medicine, Pittsburgh, PA, United States, ${ }^{8}$ University of Washington Center for AIDS \& STDs, Seattle, WA, United States; ${ }^{9}$ Columbia University, New York, NY, United States

Background Lubricants (lube) used during receptive anal intercourse (RAI) may affect the acquisition of rectally sexually transmitted infections (rSTIs). We assessed the association between lube use during RAI and rSTI among men who have sex with men (MSM).

Methods In Project Aware, a randomised controlled trial of HIV testing and counselling at 9 US Sexually Transmitted Disease clinics, in 2010 at a 6 month visit 951 MSM completed a web survey on lube use and testing for syphilis and rectal chlamydia and gonorrhoea (rSTIs/syphilis). We used multivariable logistic regression (MVLR) to assess the association of proportion of RAI using lube and rSTIs/syphilis.

Results 589 (61.9\%) of 951 men reported having receptive anal intercourse, of whom $12.9 \%(n=76)$ were diagnosed with $\mathrm{rSTI} /$ syphilis. In the prior month, men reported using lube during a mean of $83 \%$ of RAI acts (median 1.0) and condoms during $54 \%$ (median $67 \%$ ). The mean/median number of unprotected sex partners in the last 6 months (Upartners) was 2.64/1.0. Lube use was not directly associated with $\mathrm{rSTI} /$ syphilis (OR $=0.9995 \% \mathrm{CI}(.60 .1 .66)$. In MVLR adjusting for education, Upartners and condom use, the association between rSTI/syphilis and lube use increased with age and proportion of RAI with lube (interaction $\mathrm{p}=0.02$ ); among men age 32 , the lube use during $50 \%$ and $75 \%$ of RAI acts was associated with adjusted odds ratios of 3.98 (95\% CI 1.07, 14.81) and $5.03(95 \%$ CI 1.01, 25.07), respectively and risk increased at ages greater than 32

Conclusions Although these findings provide further evidence that frequent lubricant use during RAI can facilitate the acquisition of rectal STIs/syphilis among MSM it suggests the risk is only for older MSM. Further research is needed to assess differential risk by types of lubricants across age groups and provide rapid advice to the community who practise RAI on use of safe products.

\section{P3.138 INCREASING ASYMPTOMATIC LYMPHOGRANULOMA VENEREUM INFECTION IN THE UK: RESULTS FROM A NATIONAL CASE-FINDING STUDY}

doi:10.1136/sextrans-2013-051184.0597 